\title{
Perturbation Theory of Wightman Functions
}

\author{
O. Steinmann \\ Fakultät für Physik, Universität Bielefeld, W-4800 Bielefeld 1, Germany
}

Received July 27, 1992

\begin{abstract}
A perturbative expansion of the Wightman functions, and more generally of vacuum expectation values of products of time-ordered and anti-time-ordered products, is derived for $\Phi_{4}^{4}$ field theory. The result is expressed as a sum over generalized Feynman graphs. The derivation is based exclusively on the equation of motion and the Wightman axioms. Neither canonical commutation relations nor asymptotic conditions are needed at any point. In the zero-mass case the individual graphs are infrared divergent, but the sum over all graphs of a given order is convergent.
\end{abstract}

\section{Introduction}

Both the time-ordered functions ( $\tau$-functions) and the Wightman functions ( $W$ functions) of a relativistic quantum field theory contain in principle the full information on the theory. This is fortunate because functions, even generalized ones, are in many respects easier to work with than unbounded operators.

In many approximation schemes, especially in perturbation theory (PT), the $\tau$ functions are the objects which are simplest to calculate. They are also of high practical value because of their close connection with the $S$-matrix. The $W$-functions, on the other hand, are more useful for examining the basic physical structure of a theory, because their properties are very directly related to the fundamental assumptions like relativistic invariance, spectral properties, and locality. In particular, the reconstruction of the operator formulation of the theory from the $W$-functions is very transparent. This may be helpful for such problems as the exact characterization of the physical state space of a gauge theory, which problem is closely related to the confinement question. Also, the $W$-functions are not subject to the notorious ambiguities of the definition of the time-ordered products. It is therefore desirable to have systematic, if only approximate, methods of calculating $W$-functions.

PT is still one of the most powerful and best understood approximation schemes in quantum field theory. It can give significant insights into the structure of a theory 
even when it is useless from a numerical point of view, on account of the interaction strength. A systematic development of the PT of $W$-functions is therefore clearly of interest. Such a development is the subject of the present paper.

More generally, we will derive perturbative expansions for the vacuum expectation values of arbitrary products of time-ordered and anti-time-ordered products of fields. We denote the time-ordered product of the fields $\Phi\left(x_{1}\right), \ldots, \Phi\left(x_{n}\right)$, by $T^{+}\left(x_{1}, \ldots, x_{n}\right)$ or $T^{+}(X)$, where $X$ stands for the set of variables $X=$ $\left\{x_{1}, \ldots, x_{n}\right\}$. The anti-time-ordered product of these fields is called $T^{-}(X)$. The expressions to be discussed are

$$
\mathscr{W}\left(X_{1}, s_{1}\left|X_{2}, s_{2}\right| \ldots \mid X_{N}, s_{N}\right):=\left\langle 0\left|T^{s_{1}}\left(X_{1}\right) T^{s_{2}}\left(X_{2}\right) \ldots T^{s_{N}}\left(X_{N}\right)\right| 0\right\rangle,
$$

where $|0\rangle$ is the vacuum, the $s_{i}$ are signs, and the $X_{i}$ are non-overlapping finite sets of 4-vectors. $T^{+}$and $T^{-}$factors may occur in an arbitrary order. The $T^{-}$product of a number of fields is the adjoint of the $T^{+}$product of the adjoints of the original fields. Hence the definiton (1.1) includes the important case $\left\langle 0\left|T^{*}\left(X_{1}\right) T\left(X_{2}\right)\right| 0\right\rangle$ which occurs in unitarity relations, and whose Fourier transform gives a direct expression for inclusive cross sections, even in theories in which the $S$-matrix does not exist. This has been shown for QED in [1]. The $\tau$ - and $W$-functions are included in (1.1) as special cases. The perturbative representation of $\tau$ as sum over Feynman graphs is ancient lore. A graph representation of the $\mathscr{W}$-functions with $T^{+}$-factors only has been given by Ostendorf [2].

Our derivation is based on a) the equations of motion of the theory, b) the axiomatic properties of the $W$-functions [3], and c) the usual renormalization conditions. Neither equal-time commutation relations nor asymptotic conditions are used at any point.

For simplicity we consider only the example of the $\Phi_{4}^{4}$ theory. But our methods generalize immediately to more complicated renormalizable models, e.g. QED or Yang-Mills theories in covariant, local gauges. ${ }^{1}$

The $\Phi_{4}^{4}$ model is the theory of a hermitian, scalar field $\Phi(x)$ in four dimensional Minkowski space, which satisfies the Wightman axioms [3] and the equation of motion

$$
K \Phi(x)=-\frac{g}{6} N\left([\Phi(x)]^{3}\right) .
$$

Here $K$ is the Klein-Gordon operator $K=-\partial_{\mu} \partial^{\mu}-m^{2}, g$ is the coupling constant. The symbol $N$ stands for "normal product" and denotes the renormalization prescription which is needed to make sense of the a priori undefined power $\Phi^{3}$ of the distribution-valued field $\Phi$. The ultraviolet difficulties are not avoided by our method, but they can be handled by standard procedures.

The $W$-function

$$
W\left(x_{1}, \ldots, x_{n}\right)=\left\langle 0\left|\Phi\left(x_{1}\right) \ldots \Phi\left(x_{n}\right)\right| 0\right\rangle
$$

must, then, solve the differential equations

$$
K_{i} W\left(x_{1}, \ldots, x_{i}, \ldots, x_{n}\right)=-\frac{g}{6}\left\langle 0\left|\Phi\left(x_{1}\right) \ldots N\left(\Phi^{3}\left(x_{\imath}\right)\right) \ldots\right| 0\right\rangle
$$

for $i=1, \ldots, n$. Moreover, it must satisfy the following Wightman conditions.

\footnotetext{
1 An application of the method to physical gauges has already been discussed in [4], but the simpler case of local theories has never been considered in print
} 
a) Invariance:

$$
W\left(\Lambda x_{1}+a, \ldots, \Lambda x_{n}+a\right)=W\left(x_{1}, \ldots, x_{n}\right)
$$

for all orthochronous Lorentz transformations $\Lambda$ and translations $a$.

b) Reality:

$$
W\left(x_{n}, \ldots, x_{1}\right)=W\left(x_{1}, \ldots, x_{n}\right)^{*}
$$

c) Locality:

$$
W\left(\ldots, x_{i}, x_{\imath+1}, \ldots\right)=W\left(\ldots, x_{\imath+1}, x_{i}, \ldots\right) \quad \text { if } \quad\left(x_{i}-x_{\imath+1}\right)^{2}<0 .
$$

d) Spectrum: The support of the Fourier transform $\tilde{W}\left(p_{1}, \ldots, p_{n}\right)$ of $W\left(x_{1}, \ldots\right.$, $\left.x_{n}\right)$ is contained in the set $\left\{P_{i}:=\left(p_{1}+\ldots+p_{i}\right) \in \bar{V}_{+}\right.$for all $\left.i<n, \sum_{1}^{n} p_{i}=0\right\}$.

e) Cluster property:

$$
\begin{aligned}
& \lim _{a \rightarrow \infty} W\left(x_{1}, \ldots, x_{\alpha}, x_{\alpha+1}+a, \ldots, x_{n}+a\right) \\
& \quad=W\left(x_{1}, \ldots, x_{\alpha}\right) W\left(x_{\alpha+1}, \ldots, x_{n}\right)
\end{aligned}
$$

if $a$ tends to infinity in a space-like direction. From locality it follows that (1.7) holds also if the two sets $\left\{x_{1}, \ldots, x_{\alpha}\right\},\left\{x_{\alpha+1}, \ldots, x_{n}\right\}$ are not initial and final segments of $X$, but are any two complementary subsets.

Wightman positivity will not be used.

Finally, we need some normalization conditions. They are conventionally stated for the $\tau$-functions, and this is also most convenient for us. We must therefore give a definition of the function (1.1) in terms of $W$-functions. This is done by demanding that $\mathscr{W}\left(X_{1}, s_{1} \mid \ldots\right)$ satisfy the obvious invariance, reality, and spectral properties corresponding to conditions a), b) and d) for $W$, and in addition the splitting relation

$$
\mathscr{W}\left(\ldots\left|X_{1} \cup X_{2},+\right| \ldots\right)=\mathscr{W}\left(\ldots\left|X_{1},+\right| X_{2},+\mid \ldots\right)
$$

if $x_{i}^{0}>x_{j}^{0}$ for all $x_{i} \in X_{1}, x_{j} \in X_{2}$. An analogous requirement holds for $T^{-}$factors. Also, $\mathscr{W}\left(\ldots\left|X_{\imath}, s_{i}\right| \ldots\right)$ must be invariant under permutations of the elements of $X_{i}$.

Let $\tilde{\tau}\left(p_{1}, \ldots, p_{n}\right)$ be the Fourier transform of $\tau\left(x_{1}, \ldots, x_{n}\right)=\mathscr{W}(X,+), \tilde{\tau}^{t}$ its truncated $(=$ connected $)$ part, which is recursively defined by

$$
\tilde{\tau}^{t}\left(p_{1}, p_{2}\right)=\tilde{\tau}\left(p_{1}, p_{2}\right), \quad \tilde{\tau}^{t}\left(p_{1}, P\right)=\tilde{\tau}\left(p_{1}, P\right)-\sum_{P_{1} \cup P_{2}=P} \tilde{\tau}\left(p_{1}, P_{1}\right) \tilde{\tau}^{t}\left(P_{2}\right)
$$

with $P=\left\{p_{2}, \ldots, p_{n}\right\}, n>2$. The sum extends over all partitions of $P$ into two complementary, non-empty, subsets $P_{1}, P_{2}$.

By translation invariance these functions are of the form

$$
\tilde{\tau}^{(t)}\left(p_{1}, \ldots, p_{n}\right)=\delta^{4}\left(\sum p_{i}\right) \hat{\tau}^{(t)}\left(p_{1}, \ldots, p_{n-1}\right) .
$$

In the massive case $m>0$ we demand that the 2-point function is in a neighbourhood of the mass shell $p^{2}=m^{2}$ given by (we use units with $\hbar=c=1$ )

$$
\hat{\tau}(p)=\frac{i}{2 \pi} \frac{1}{p^{2}-m^{2}+i \varepsilon}+\text { regular },
$$


and that the amputated, truncated, 4-point function

$$
\tilde{\tau}^{\prime}\left(p_{1}, \ldots, p_{4}\right)=\prod_{1}^{4}\left(p_{i}^{2}-m^{2}\right) \tilde{\tau}^{t}\left(p_{1}, \ldots, p_{4}\right)
$$

satisfy

$$
\hat{\tau}^{\prime}(0,0,0)=-i g
$$

Condition (1.10) can easily be reformulated in terms of the 2-point $W$-function. It means that $\hat{W}(p)$, defined in analogy to (1.9), is given by

$$
\hat{W}(p)=\theta\left(p_{0}\right) \delta\left(p^{2}-m^{2}\right)
$$

in a neighbourhood of the mass shell.

The conditions $(1.10-11)$ cannot be used in the case $m=0$, due to infrared (IR) problems. In that case we generalize (1.11) to

$$
\hat{\tau}^{\prime}\left(\mu_{1}, \mu_{2}, \mu_{3}\right)=-i g
$$

where $\left(\mu_{1}, \ldots, \mu_{4}\right)$ is a "symmetry point" characterized by $\left(\mu_{i}, \mu_{\jmath}\right)=\frac{1}{3} \mu^{2}\left(4 \delta_{i j}-1\right)$, $\mu^{2}<0$. Due to Lorentz invariance, the propagator $\hat{\tau}(p)$ is a function of $p^{2}$ only. We demand that $\hat{\tau}\left(p^{2}\right)$ still have a 1-particle singularity at the origin, which is now not a pole but must be defined as a distribution. The same shall hold in every finite order of PT. Furthermore, the normalization condition

$$
\left.\frac{d}{d p^{2}}\left[\left(p^{2}\right)^{2} \hat{\tau}\left(p^{2}\right)\right]\right|_{p^{2}=\mu^{2}}=\frac{i}{2 \pi}
$$

shall be satisfied.

Finally we require that $\hat{\tau}\left(p^{2}\right)$ tend to zero for $p^{2} \rightarrow \infty$. This condition is necessary for ensuring renormalizability and is also an, usually tacit, assumption of the conventional formalism.

The arbitrariness of these normalization conditions gives rise to the concept of the renormalization group, which will, however, not be discussed in this paper. An important restriction exists in theories with more than one field. Consider e.g. the $\Phi_{1}^{2} \Phi_{2}^{2}$ model, where the $\Phi_{\alpha}$ are two scalar fields with masses $m_{\alpha}$. Define $K_{\alpha}=-\left(\square+m_{\alpha}^{2}\right)$. Then the consistency condition

$$
\begin{aligned}
& K_{1}(x)\left\langle 0\left|\ldots \Phi_{1}(x) \ldots N\left(\Phi_{1}^{2}(y) \Phi_{2}(y)\right) \ldots\right| 0\right\rangle \\
& \quad=K_{2}(y)\left\langle 0\left|\ldots N\left(\Phi_{1}(x) \Phi_{2}^{2}(x)\right) \ldots \Phi_{2}(y) \ldots\right| 0\right\rangle
\end{aligned}
$$

must be satisfied.

The absence of canonical commutation relations raises the question of the status of Planck's constant in the formalism. It can be answered by means of the fundamental relation

$$
\left[P_{\mu}, \Phi(x)\right]=-i \hbar \partial_{\mu} \Phi(x),
$$

where the $P_{\mu}$ are the energy-momentum operators. We can fix the energy scale by demanding $P_{\mu}|0\rangle=0$ and prescribing the value of $P_{0}$ on a (improper) reference eigenstate $\Psi$ of $P_{0}$, which is uniquely identifiable both in the theory and in the laboratory. In a theory with massive particles we can choose $\Psi$ to be a 1-particle state in its rest frame. If only massless particles exist, we can fix $\Psi$ to be a 1particle state with a prescribed wave vector $\mathbf{k}$. This is measurable in interference 
experiments and is theoretically constructible by means of the Fourier transformed field $\hat{\Psi}(k)=\int d^{4} x e^{i k x} \Phi(x)$. This definition can also be extended to infraparticles. $\hbar$ is then determined by evaluating the matrix elements of both sides of Eq. (1.16) between $\Psi$ and the vacuum, assuming that $\Psi$ is chosen such that $\langle\Psi|\Phi(x)| 0\rangle \neq 0$. Alternatively, if we fix the numerical value of $\hbar$ by decree (e.g. $\hbar=1$ ), then (1.16) determines the energy scale.

In PT the equations of motion (1.3) become

$$
K_{i}\left\langle 0\left|\ldots \Phi\left(x_{i}\right) \ldots\right| 0\right\rangle_{\sigma}=-\frac{g}{6}\left\langle 0\left|\ldots N\left(\Phi\left(x_{i}\right)^{3}\right) \ldots\right| 0\right\rangle_{\sigma-1}
$$

where $\langle\ldots\rangle_{\sigma}$ is the $g^{\sigma}$ term in the perturbative expansion of $\langle\ldots\rangle$. These equations can be solved by induction with respect to $\sigma$, starting from the free case $\sigma=0$, where the right-hand side is zero. For $\sigma>0$ the right-hand sides can be calculated from the known solution in order $\sigma-1$, assuming that the renormalization prescription $N$ has been unambiguously fixed. $W_{\sigma}$ is then obtained as solution of a system of linear differential equations supplemented by the conditions indicated above. In Sect. 2 it will be shown that this procedure leads to a unique solution. In Sect. 3 a formal, unrenormalized solution will be written down as a sum over generalized Feynman graphs. Its ultraviolet divergences are removed in Sect. 4 by the BPHZ method, which we reformulate in $x$-space. In Sect. 5 existence of our expressions also in the IR case $m=0$ will be shown. This is important for possible applications of the formalism to gauge theories.

\section{Uniqueness}

Assuming the $W$-functions to be know up to order $\sigma-1$, we find $W_{\sigma}$ as a solution of Eq. (1.17) satisfying the Wightman conditions a)-e) and the normalization conditions as stated in Sect.1. We want to show that $W_{\sigma}$ is determined uniquely by these conditions, if it exists at all.

Let $W_{\sigma}^{1}, W_{\sigma}^{2}$, be two solutions of the problem. Then their difference

$$
h\left(x_{1}, \ldots, x_{n}\right)=W_{\sigma}^{1}\left(x_{1}, \ldots, x_{n}\right)-W_{\sigma}^{2}\left(x_{1}, \ldots, x_{n}\right)
$$

satisfies the homogeneous equations

$$
K_{i} h\left(\ldots, x_{i}, \ldots\right)=0
$$

and the conditions a)-e).

The 2-point function $\hat{h}(p)$, defined as in (1.9), has its support in $\left\{p^{2} \geq 0, p^{0} \geq 0\right\}$, and is there a function of $p^{2}$ only. Equation (2.2) becomes $\left(p^{2}-m^{2}\right) \hat{h}=0$, so that

$$
\hat{h}\left(p^{2}\right)=c \delta\left(p^{2}-m^{2}\right)
$$

with $c$ a real constant. For $m>0$ we find from (1.12) that $\hat{h}\left(p^{2}\right)$ vanishes at $p^{2}=m^{2}$, hence $c=0$ and thus $h=0$. If $m=0$ we form from $h\left(x_{1}, x_{2}\right)$ the corresponding time-ordered function $h_{\tau}$ and its $p$-space form $\hat{h}_{\tau}\left(p^{2}\right)$. From (2.3) and the vanishing of $\hat{h}_{\tau}$ at infinity we find that $\hat{h}_{\tau}=\frac{i}{2 \pi} c\left(p^{2}+i \varepsilon\right)^{-1}$. Condition (1.14), which must hold for both $W^{1}$ and $W^{2}$, again yields $c=0$. 
For $n>2$ we show first that $h\left(x_{1}, \ldots, x_{n}\right)$ is invariant under permutations of its arguments. Define

$$
\Delta\left(\ldots, x_{i}, x_{i+1}, \ldots\right)=h\left(\ldots, x_{\imath}, x_{\imath+1}, \ldots\right)-h\left(\ldots, x_{\imath+1}, x_{\imath}, \ldots\right)
$$

From (2.2) it follows that the Fourier transform $\tilde{\Delta}\left(\ldots, p_{i}, p_{i+1}, \ldots\right)$ contains the factors $\delta\left(p_{i}^{2}-m^{2}\right) \delta\left(p_{\imath+1}^{2}-m^{2}\right)$. We replace the variables $p_{\imath}, p_{i+1}$, by $P=\frac{1}{2}\left(p_{i}+p_{i+1}\right)$, $Q=\frac{1}{2}\left(p_{\imath}-p_{\imath+1}\right)$, which are conjugate to $X=x_{i}+x_{\imath+1}, \xi=x_{i}-x_{\imath+1}$. The support of $\tilde{\Delta}$ consists then of four components defined by

$$
P_{0}+Q_{0}= \pm \omega(\mathbf{P}+\mathbf{Q}), \quad P_{0}-Q_{0}= \pm \omega(\mathbf{P}-\mathbf{Q}),
$$

with $\omega(\mathbf{p})=\left(\mathbf{p}^{2}+m^{2}\right)^{1 / 2}$. Adding the two conditions we find

$$
2 P_{0}= \pm \omega(\mathbf{P}+\mathbf{Q}) \pm \omega(\mathbf{P}-\mathbf{Q})
$$

independently of $Q_{0}$. For a fixed $P \neq 0$ neither of these four relations (corresponding to the sign alternatives) is satisfied identically in $Q$ : there exists an open neighbourhood in $Q$-space in which $\tilde{\Delta}$ vanishes identically.

Locality demands that $\Delta(\ldots, X, \xi, \ldots)$ vanish for $\xi^{2}<0$. Hence the support of $\Delta_{ \pm}=\theta\left( \pm \xi_{0}\right) \Delta(\ldots, X, \xi, \ldots)$ is contained in the half-cone $\bar{V}_{ \pm}$. The Fourier transform

$$
\tilde{\Delta}_{ \pm}= \pm i \int d u \frac{1}{Q_{0}-u \pm i \varepsilon} \tilde{\Delta}(\ldots, P, u, \mathbf{Q}, \ldots)
$$

is as a function of $Q$ a boundary value of an analytic function in the tube $\mathscr{T}_{ \pm}=$ $\left\{Q: \operatorname{Im} Q \in V_{ \pm}\right\}$[3]. But the support condition (2.5), being independent of $Q_{0}$, holds also for $\tilde{\Delta}_{ \pm}$. Hence we find by analytic continuation that $\tilde{\Delta}_{ \pm} \equiv 0$, and thus also $\tilde{\Delta}=\tilde{\Delta}_{+}+\tilde{\Delta}_{-} \equiv 0$ for $P \neq 0$. Assuming that the vanishing of $h$ has already been shown in all lower orders and in order $\sigma$ for fewer than $n$ variables, we find from the cluster property that

$$
\lim _{\mathbf{a} \rightarrow \infty} h\left(x_{1}, \ldots, x_{\imath}+\mathbf{a}, x_{i+1}+\mathbf{a}, \ldots\right)=W_{0}\left(x_{\imath}, x_{i+1}\right) h\left(\ldots, \hat{x}_{\imath}, \hat{x}_{\imath+1}, \ldots\right)=0 .
$$

The same relation holds if the two variables change place, hence it holds for $\Delta$. This excludes the possibility of $\tilde{\Delta}$ being a sum of terms containing a factor $\delta^{4}(P)$ or derivatives thereof. We have thus shown that $\Delta \equiv 0$, i.e. that $h$ is invariant under the exchange of any two adjacent variables. This proves the total symmetry of $h$.

In particular this implies

$$
\tilde{h}\left(p_{1}, \ldots, p_{n-1}, p_{n}\right)=\tilde{h}\left(p_{n}, p_{1}, \ldots, p_{n-1}\right) .
$$

But by the spectrum condition the left-hand side has its support contained in $p_{n} \in \bar{V}_{-}$, the right-hand side in $p_{n} \in \bar{V}_{+}$, and these two supports intersect only at $p_{n}=0$. This point support is again excluded by the cluster property and the vanishing of the 1-point function.

We have thus proved that $h \equiv 0$ for all $n$ and $\sigma$, i.e. that our conditions indeed fix the theory uniquely. 


\section{Unrenormalized Solution}

We first write down the rules for calculating the unrenormalized $\mathscr{W}_{\sigma}\left(X_{1}, s_{1}\right)$ $\left.\ldots \mid X_{N}, s_{N}\right)$, and then justify these rules by showing that they satisfy all the necessary requirements, apart from the normalization conditions $(1.10-14)$ which are intimately connected with renormalization and will be dealt with in the following section. The rules given here have already been stated without proof in an appendix of [5].

$\mathscr{W}_{\sigma}$ is represented as a sum over generalized Feynman graphs which are defined as follows. Draw first an ordinary Feynman graph of the $\Phi^{4}$ theory, with $\left|X_{i}\right|$ external and $\sigma$ internal vertices. Here $|X|$ is the number of elements of the set $X$. The graph need not be connected, but it must not contain any components without external points (vacuum-vacuum graphs). Also, there are no lines connecting a vertex to itself. This graph is called the "scaffolding" of the generalized graph. ${ }^{2}$ It is partitioned into non-overlapping subgraphs, called "sectors," such that the external points of an $X_{2}$ belong all to the same sector, but variables of different $X_{i}$ to different sectors. These $X_{2}$-sectors are called "external." In general there exist also "internal" sectors not containing external points. To each sector $S$ we affix a number $\nu(S)$ according to the following rules:

i) $\nu(S)=i$ for the external $X_{i}$ sector.

ii) For an internal sector $\nu(S)$ is non-integer and lies between the maximal and the minimal number of the adjacent sectors, i.e. of the sectors which are directly linked to $S$ by a line of the scaffolding.

iii) The sectors are either $T^{+}$or $T^{-}$sectors. The external sector with number $i$ is a $T^{s_{i}}$ sector. An internal sector with $i<\nu(S)<i+1$ is a $T^{s_{2}}$ sector if $s_{i}=s_{i+1}$. If $s_{\imath} \neq s_{i+1}$ there are no internal sectors with $i<\nu(S)<i+1$.

Two partitions with the same topology are only considered different if for at least one pair of sectors $S, S^{\prime}$, we have $\nu(S)>\nu\left(S^{\prime}\right)$ in one case, $\nu(S)<\nu\left(S^{\prime}\right)$ in the other case.

With a partitioned graph we associate a Feynman integrand as follows. To the external points correspond the variables $x_{i}$. To each internal vertex we assign an integration variable $u_{\jmath}, j=1, \ldots, \sigma$. We use the notation $z_{\imath}, z_{j}, \ldots$ for variables which may be either external or internal. Within a $T^{+}$sector we apply the usual Feynman rules: each internal vertex carries a factor $-i g$, a line connecting the vertices $z_{\imath}$ and $z_{j}$ carries the propagator $-i \Delta_{F}\left(z_{i}-z_{j}\right)$. Within a $T^{-}$sector the complexconjugates of these rules apply. A line connecting two vertices $z_{i}, z_{j}$, in different sectors, with $z_{i}$ belonging to the sector with the lower number, carries the propagator $-i \Delta_{+}\left(z_{i}-z_{j}\right)$. Each internal sector contributes a factor -1 . Finally, the graph must be divided by the usual symmetry number, if it is invariant under certain permuatitons of points and lines.

$\mathscr{W}_{\sigma}^{\circ}$ is the sum over all partitioned graphs of order $\sigma$ integrated over the internal variables.

The field $\Phi(x)$ can be considered to be either a time-ordered or an anti-time-ordered product with one factor. Hence we have

$$
W_{\sigma}\left(x_{1}, \ldots, x_{n}\right)=\mathscr{W}_{\sigma}\left(x_{1}, \pm\left|x_{2}, \pm\right| \ldots \mid x_{n}, \pm\right) .
$$

2 The scaffolding was called "skeleton" in [5]. This was an unfortunate choice, since "skeleton" is already used with a different meaning in the theory of Feynman graphs 
This means that our rules give $2^{n}$ different representations for $W_{\sigma}$. We must show that the result is independent of the choice of the $n$ signs. To this end we state a lemma which is also useful for other purposes, e.g. for proving unitarity.

Lemma 3.1. Let $\Gamma$ be a subgraph with $N$ vertices, including $E$ external points, of a scaffolding, with variables $Z=\left\{z_{1}, \ldots, z_{N}\right\}$. Let $\Gamma_{L} \cup \Gamma_{R}=\Gamma$ be any partition of $\Gamma$ into a $T^{+}$sector $\Gamma_{L}$ and a $T^{-}$sector $\Gamma_{R}, \nu\left(\Gamma_{L}\right)<\nu\left(\Gamma_{R}\right)$, with $Z_{L}$ and $Z_{R}$ the corresponding subsets of $Z$. Let $I_{L R}$ be the integrand of this partitioned subgraph. Then

$$
\sum_{\Gamma_{L}}(-1)^{E_{L}} I_{L R}(Z)=0
$$

with $E_{L}$ the number of external points in $\Gamma_{L}$.

This is a well-known result (see e.g. [6, Sect. II.6] or [7, Chap. 6]). Nevertheless it is useful for later reference to give a short proof. Consider a particular partition $\Gamma_{L, R}$. Let $L$ be the number of vertices in $\Gamma_{L}$. Because of $\Delta_{F}(\xi)=\Delta_{+}(\xi)$ for $\xi^{0} \geq 0$, $\overline{\Delta_{F}(\xi)}=-\Delta_{+}(\xi)$ for $\xi^{0} \leq 0$ we can write the corresponding contribution to (3.2) as

$$
\begin{aligned}
& i^{N-E} g^{N-E}(-1)^{L} \sum_{P} \sum_{Q} \theta\left(z_{1}^{P}-z_{2}^{P}\right) \ldots \theta\left(z_{L-1}^{P}-z_{L}^{P}\right) \\
& \quad \times\left[\theta\left(z_{L}^{P}-z_{L+1}^{Q}\right)+\theta\left(z_{L+1}^{Q}-z_{L}^{P}\right)\right] \\
& \quad \times \theta\left(z_{L+2}^{Q}-z_{L+1}^{Q}\right) \ldots \theta\left(z_{N}^{Q}-z_{N-1}^{Q}\right)\left\{z_{1}^{P} \ldots z_{L}^{P} z_{L+1}^{Q} \ldots z_{N}^{Q}\right\} .
\end{aligned}
$$

Here we have defined $\theta(\xi):=\theta\left(\xi^{0}\right)$. The summations extend over all permutations $\left(z_{1}^{P}, \ldots, z_{L}^{P}\right)$ of $Z_{L}$ and $\left(z_{L+1}^{Q}, \ldots, z_{N}^{Q}\right)$ of $Z_{R}$, and $\left\{z_{1}^{P} \ldots z_{N}^{Q}\right\}$ is the product over all lines in $\Gamma$ of the propagators $i \Delta_{+}\left(z_{\imath}-z_{j}\right)$ with $z_{i}$ standing to the left of $z_{j}$ in $\{\ldots\}$. Take the contribution of a particular pair $(P, Q)$ and the second term in the square bracket:

$$
i^{N-E} g^{N-E}(-1)^{L} \ldots \theta\left(z_{L-1}^{P}-z_{L}^{P}\right) \theta\left(z_{L+1}^{Q}-z_{L}^{P}\right) \theta\left(z_{L+2}^{Q}-z_{L+1}^{Q}\right) \ldots\left\{z_{1}^{P} \ldots z_{N}^{Q}\right\} .
$$

This is cancelled by the contribution from the first term in [...] and the same $\{\ldots\}$ in the partition $Z_{L}^{\prime}=Z_{L} \cup z_{L+1}^{Q}, Z_{R}^{\prime}=Z_{R} \backslash z_{L+1}^{Q}$. If $\Gamma_{L}=\Gamma$ or $\emptyset$, then there is no square bracket, but there are nevertheless $N-1 \theta$-factors present, and cancellation happens as before against a term with $\Gamma_{R}=x_{N}$ or $\Gamma_{L}=x_{1}$ respectively.

Note that (3.2) remains correct if we sum over the partitions with $\nu\left(\Gamma_{L}\right)>\nu\left(\Gamma_{R}\right)$ instead of the reverse ordering, as can be shown in the same way.

For the proof of equivalence of the various $W$-representations we need the lemma for the cases that $\Gamma$ contains one or no external point. We use the following abbreviated notation: $S_{x}$ denotes the integrand of a $T^{+}$sector containing the external variable $x$, $S$ denotes the integrand of an internal $T^{+}$sector, $\bar{S}_{x}$ and $\bar{S}$ correspond to $T^{-}$sectors. A product $S^{m} \bar{S}_{x} S^{n}$, or the like, stands for

$$
S^{m} \bar{S}_{x} S^{n}=\sum S\left(U_{1}\right) \ldots S\left(U_{m}\right) \bar{S}_{x}\left(U_{m+1}\right) S\left(U_{m+2}\right) \ldots S\left(U_{m+n+1}\right),
$$

where the sum extends over all partitions of the set $U$ of internal vertices of $\Gamma$ into $(m+n+1)$ subsets. $U_{m+1}$ may be empty, but not the other $U_{\imath}$. All propagators linking points of $\Gamma$ are included, also those connecting different factors. The relevant cases of (3.2) read then

$$
\bar{S}_{x}=S_{x}+S_{x} \bar{S}-S \bar{S}_{x}, \quad \bar{S}=-S-S \bar{S} .
$$


Define

$$
A_{m, n}=(-1)^{m+n} S^{m} S_{x} S^{n} \bar{S}, \quad B_{m}=(-1)^{m} S^{m} \bar{S}_{x}
$$

Equation (3.3) yields

$$
\begin{aligned}
A_{m, n} & =A_{m, n+1}+(-1)^{m+n+1} S^{m} S_{x} S^{n+1}, \\
B_{m} & =B_{m+1}+A_{m, 0}+(-1)^{m} S^{m} S_{x} .
\end{aligned}
$$

Starting from $\bar{S}_{x}=B_{0}$ and iterating (3.4) as often as necessary, we obtain

$$
\bar{S}_{x}=\sum_{m, n}(-1)^{m+n} S^{m} S_{x} S^{n} .
$$

This proves

$$
\mathscr{W}_{\sigma}\left(\ldots\left|x_{\imath},+\right| x,-\left|x_{j},+\right| \ldots\right)=\mathscr{W}_{\sigma}\left(\ldots\left|x_{i},+\right| x,+\left|x_{j},+\right| \ldots\right),
$$

i.e. $s_{x}=-$ can be replaced by $s_{x}=+$ in $W_{\sigma}$, if the $x$-sector stands between two $T^{+}$sectors. In the same way one shows that this replacement is possible between two $T^{-}$sectors.

In the case $\left|x_{i},+\right| x,-\left|x_{\jmath},-\right|$ we proceed similarly, defining

$$
C_{m, n}=(-1)^{m+n} S^{m} S_{x} \bar{S}^{n}, \quad \bar{C}_{m, n}=(-1)^{m+n} S^{m} \bar{S}_{x} \bar{S}^{n}
$$

We need to show that

$$
\sum_{n} \bar{C}_{0, n}=\sum_{m} C_{m, 0}
$$

From (3.3) we obtain

$$
\bar{C}_{m, n}=C_{m, n}-C_{m, n+1}+\bar{C}_{m+1, n} .
$$

Repeated application of this relation to the left-hand side of (3.6) yields the desired relation:

$$
\begin{aligned}
\sum_{n} \bar{C}_{0, n} & =\sum_{n}\left(C_{0, n}-C_{0, n+1}\right)+\sum_{n} \bar{C}_{1, n}=C_{0,0}+\sum_{n} \bar{C}_{1, n} \\
& =C_{0,0}+\sum_{n}\left(C_{1, n}-C_{1, n+1}\right)+\sum_{n} \bar{C}_{2, n}=C_{0,0}+C_{1,0}+\sum_{n} \bar{C}_{2, n} \\
& \ldots \ldots \ldots \ldots \\
& =\sum_{m} \bar{C}_{m, 0} .
\end{aligned}
$$

The case $|-|+|+|$ its treated analogously.

We will now show that our expressions for $\mathscr{W}_{\sigma}$ and $W_{\sigma}$ satisfy all necessary requirements. The splitting relation (1.8) has been proved by Ostendorf [2] for the special case that all $s_{i}$ are positive. The generalization of this proof to our more complicated situation is straightforward.

Next we check the equations of motion (1.17). The Klein-Gordon operator $K_{i}$ annihilates all graphs contributing to $W_{\sigma}\left(\ldots, x_{\imath}, \ldots\right)$ in which the $x_{i}$-point is an endpoint of a $\Delta_{+}$-line, i.e. those in which $x_{i}$ is either a sector by itself or is not directly connected to the rest of its sector. $K_{i}$ applied to the remaining graphs gives precisely the graphs contributing to the right-hand side $-\frac{g}{6}\left\langle 0\left|\ldots \Phi\left(x_{\imath}\right)^{3} \ldots\right| 0\right\rangle_{\sigma-1}$, 
provided that the product $\Phi^{3}$ occurring in here is a $T^{+}$or a $T^{-}$product, depending on whether we choose $s_{2}$ to be + or - . In the original Eq. (1.2), $\Phi^{3}$ was meant to be an ordinary product. However, these three possibilities actually coincide. In order to see this, we define $\phi^{3}$ by point splitting, i.e. we cut the $N$-vertex in the right-hand side open, and consider $\left\langle 0\left|\ldots \Phi\left(y_{1}\right) \Phi\left(y_{2}\right) \Phi\left(y_{3}\right) \ldots\right| 0\right\rangle_{\sigma-1}$ in the limit $y_{1,2,3} \rightarrow x_{2}$. Take the various possible orderings of the $\Phi\left(y_{r}\right)$ : ordinary product in any order, $T^{+}$ product, $T^{-}$product. The difference between any two of these orderings is a sum over terms $\left[\Phi\left(y_{r}\right), \Phi\left(y_{s}\right)\right] \Phi\left(y_{t}\right)$ or similar, containing at least one commutator, multiplied with a product of $\theta$-functions. Hence, if the commutator $\left[\Phi\left(y_{r}\right), \Phi\left(y_{s}\right)\right]$ can be shown to vanish for $y_{r}=y_{s}=x_{\imath}$, all the orderings coincide for identical arguments. Now, according to our graph rules there exist no lines connecting $y_{r}$ with $y_{s}$ directly. This is part of the usual $N$-product definition (for free fields it amounts simply to Wick ordering). It means that the integrand of a graph contributing to the $\Phi\left(y_{r}\right) \Phi\left(y_{s}\right)$ part of the commutator is after the identification $y_{r}=y_{s}=x_{\imath}$ still well defined as a singular function of $x_{i}$ and the remaining variables, which obviously does not depend on the ordering of the $\Phi\left(y_{i}\right)$, so that we obtain complete cancellation for the commutator. The individual integrands are possibly not defined as distributions where $x_{\imath}$ coincides with one of the other variables. But this is part of the UV problem which we yet disregard. In any case, the problem disappears in the difference of graphs which we consider.

The proof of the Wightman properties a)-e) of Sect. 1 can be taken over from Ostendorf [2], except that the proof of the reality condition (1.5) is now much simplified: it is trivial to see that

$$
\mathbb{W}_{\sigma}\left(x_{1},+|\ldots| x_{n},+\right)^{*}=\mathscr{W}_{\sigma}\left(x_{n},-|\ldots| x_{1},-\right),
$$

which proves (1.5), since $W_{\sigma}$ is independent of the choice of signs $s_{\imath}$.

\section{Renormalization}

The normalization conditions (1.10-11) and (1.13-14) are formulated in momentum space, and the removal of UV divergences is easier to discuss in that space. The graph rules of Sect. 3 are easily transcribed into $p$-space, in complete analogy to the wellknown case of the $\tau$-functions. We obtain in $T^{ \pm}$sectors the vertex factors $\mp i(2 \pi)^{4} g$, and the propagators $\pm i(2 \pi)^{-4}\left(p^{2}-m^{2} \pm i \varepsilon\right)^{-1}$. The propagators of lines connecting two sectors are $(2 \pi)^{-3} \theta\left(p_{0}\right) \delta\left(p^{2}-m^{2}\right)=:(2 \pi)^{-3} \delta_{+}(p)$, the momentum $p$ flowing from the lower-numbered sector to the higher-numbered one. For each external point there is an additional factor $(2 \pi)^{3 / 2}$. Momentum is conserved in each vertex. Otherwise, the rules are the same as in $x$-space.

With respect to the UV problem we note first that loop integrals over loops extending over more than one sector are UV convergent, because the $\delta_{+}$-factors of the integrand restrict the loop variable to a compact set [2]. Hence divergences are restricted to the individual sectors, where they can be handled by standard procedures. We will use the BPHZ method, as explained e.g. in Itzykson-Zuber [8, Sect. 8-2], or in much more detail in Zimmermann [6].

We consider first the massive case $m>0$. The renormalization parts of a partitioned graph are the proper 1-particle irreducible subgraphs with two or four external (to the subgraph) lines, which are wholly contained in a single sector. The superficial degree of divergence is 0 in the 4-line case, 2 in the 2 -line case. We must, then, subtract from the integrand of a 4-line renormalization part its value at 
vanishing external momenta, and in the 2-line case we subtract the Taylor expansion up to second order in the external variables of the integrand. If these subtractions for all renormalization parts of a sector are combined according to Zimmermann's forest formula, then the sector becomes UV finite. And if this is done for all the sectors of a graph, the graph becomes finite. The result does not yet satify the normalization conditions (1.10) and (1.11). But this can then be achieved by finite renormalizations, i.e. by multiplying the 4-line vertex factor with an appropriately chosen finite renormalization constant $Z(g)$, with $Z(0)=1$, and introducing two new 2-line vertices with vertex factors $\pm i(2 \pi)^{4} \delta m^{2}(g)$ and $\pm i(2 \pi)^{4} \zeta(g)\left(p^{2}-m^{2}\right)$, where again $\delta m^{2}$ and $\zeta$ are finite functions of $g$, with $\delta m^{2}(0)=\zeta(0)=0 . Z, \delta m^{2}$, and $\zeta$, can be determined order by order from the normalization conditions.

There is a problem concerning self-energy parts, i.e. renormalization parts with two external lines. Consider such a subgraph in a $T^{+}$sector, with value $\Sigma(p)$, one of whose external lines is a $\delta_{+}$line. We have then the apparently singular product $\left(p^{2}-m^{2}+i \varepsilon\right)^{-1} \Sigma(p) \delta_{+}(p)$. However, if $\Sigma$ is properly renormalized, including the finite $\delta m^{2}$ and $\zeta$ renormalizations, then it contains the factor $\left(p^{2}-m^{2}\right)^{2}$, and the product in question vanishes, no matter in what order the factors are multiplied.

In the case $m=0$ we cannot subtract renormalization parts at vanishing external momenta, because this would lead to IR divergences. We therefore subtract the integrand in the 4-line case at the symmetry point $\left\{\mu_{\imath}\right\}$ introduced in connection with condition (1.13). In the 2-line case we subtract at first at the space-like momentum $\mu$. It is convenient, however, to change this prescription to the following, by means of finite renormalizations. Let $\bar{R}_{\Gamma}(p, \ldots)$ be the integrand of the renormalization part $\Gamma$, already subtracted for its divergent subgraphs, with $p$ its external momentum. Then we define the subtraction $T_{\Gamma}$ by

$$
\begin{aligned}
T_{\Gamma} \overline{\mathscr{R}}_{\Gamma}(p, \ldots)= & \overline{\mathscr{R}}_{\Gamma}(0, \ldots)+\left.p^{\mu} \partial_{\mu} \overline{\mathscr{R}}_{\Gamma}(p, \ldots)\right|_{p=0} \\
& +\left.\frac{1}{2} p^{\mu} p^{\nu} \partial_{\mu} \partial_{\nu} \cdot \overline{\mathscr{R}}_{\Gamma}(p, \ldots)\right|_{p=\mu} .
\end{aligned}
$$

This is still IR finite. It again yields UV convergence of $\Gamma$. Moreover, the prescription (4.1) ensures the distributionality of the singularity at $p^{2}=0$. But the last term in (4.1) destroys Lorentz invariance. The integral $A_{\Gamma}$ over the internal variables of $\mathscr{R}_{\Gamma}=\left(1-T_{\Gamma}\right) \bar{R}_{\Gamma}$ is a function of $p$ and $\mu$ which is invariant under simultaneous Lorentz transformation of $p$ and $\mu$, but not under transformation of $p$ alone: it depends on the variables $p^{2}$ and $(p, \mu)\left(\mu^{2}\right.$ is a constant). But the dependence on $(p, \mu)$ is polynomial. To see this we treat $A_{\Gamma}(p)$ as a distribution. The subtraction term (4.1) does not contribute to the integral over test functions $\varphi(p)$ whose moments up to second order vanish (we can integrate first over $p$ and then only over the internal variables). This is the case for $\varphi$ which are third derivatives of test functions. Hence a $\mu$-dependence of a third derivative of $A_{\Gamma}$ can only come from earlier subtractions performed in the interior of $\Gamma$. This can only be a dependence on the invariant $\mu^{2}$, if we assume the earlier subtractions to have been properly invariantized. Hence we find $A_{I}(p, \mu)=A_{0}\left(p^{2}\right)+a_{1}(p, \mu)+a_{2}(p, \mu)^{2}$ with constant coefficients $a_{1,2}$. The $a$-terms can be removed by finite renormalizations. Finally, condition (1.14) can be satisfied by yet another finite renormalization analogous to the $\zeta$-term in the massive case. The problem of self-energy insertions next to a sector boundary cannot be handled as simply as in the massive case, because near the mass shell $\Delta(p)$ behaves now like $p^{2} \log ^{n} p^{2}$, which does not vanish strongly enough to ensure existence of the product 
$\left(p^{2}\right)^{-1} \Sigma(p) \delta_{+}(p)$. But the ensuing singularities cancel between several graphs, which cancellation is part of the general IR cancellation to be discussed in Sect. 5 .

In order to see that the proofs given in Sect. 3 are not invalidated by renormalization, we must transform the renormalization procedure into $x$-space. This will also be useful for the following section, and it is of considerable interest in itself. The new vertices due to finite renormalizations are easily transcribed: a vertex factor $\pm i(2 \pi)^{4} C$ in $p$-space becomes $\pm i C$ in $x$-space, and the vertex factor $\pm i(2 \pi)^{4} C p_{\mu} p_{\nu}$ in a twoprong vertex becomes $\pm i C \overleftarrow{\partial_{\mu}} \vec{\partial}_{\nu}$, where the arrows over the derivatives signify that the derivatives act each on one of the propagators joining the vertex.

$\mathrm{BPHZ}$ renormalization follows exactly the same line as in $p$-space. Only the Taylor operator $T_{\Gamma}$ must be reformulated. Let $\Gamma$ be a renormalization part of the graph $G$, and let $u_{1}, \ldots, u_{N}$, be the variables of its exterior vertices, i.e. the vertices directly connected to the complementary graph $G \backslash \Gamma=: G_{\Gamma}$. Let us assume for the moment that $\Gamma$ is primitively divergent. Then the integral $I\left(u_{1}, \ldots, u_{N}\right)$ over the interior variables exists (see the following section for the handling of possible IR divergences) but is non-integrable in the sense of distributions at $u_{1}=\ldots=u_{N}$ ). Let $E\left(u_{1}, \ldots, u_{N}\right)$ be the part of the $G$-integrand coming from $G_{\Gamma}$, including the propagators of the lines connecting $\Gamma$ to $G_{\Gamma} . E$ depends also on the vertex variables of $G_{\Gamma}$, but these are not important for our prescription. Contrary to $p$-space, subtractions are now aplied to $E$, not to $I$. Define

$$
U=\frac{1}{N} \sum u_{\imath}, \quad \delta_{i}=u_{i}-U
$$

and let $\left\{\mu_{\imath}^{\varrho}\right\},\left\{\nu_{i}\right\}$ be sets of space-like 4-vectors with $\sum_{\imath} \mu_{i}^{\varrho}=\sum_{i} \nu_{\imath}=0$. The index $\varrho$ refers to the order of subtraction. For massive theories we choose $\mu_{i}^{\varrho}=\nu_{i}=0$. In the massless case we choose $\nu_{i}=\mu_{i}^{0}=\mu_{i}$ for $N=4, \nu_{i}=0$ and $\mu_{\imath}^{0,1}=0, \mu_{1}^{2}=-\mu_{2}^{2}=\mu$ for $N=2$. $\mu_{\imath}$ and $\mu$ are the vectors defined in Sect. 3. In generalizations to theories other than $\Phi_{4}^{4}$ it is important that the $\nu_{i}$ should be independent of the order of subtraction and that $\mu_{\imath}^{\varrho}=\nu_{\imath}$ except possibly for the highest $\varrho$ occurring in a given subtraction.

We define

$$
\begin{aligned}
T_{\Gamma} E\left(u_{1}, \ldots, u_{N}\right)= & \sum_{\varrho=0}^{s} \frac{1}{\varrho !} \exp \left(-i \sum_{j} \mu_{\jmath}^{\varrho} u_{j}\right) \sum_{i_{1}, \ldots, i_{\varrho}=1}^{N} \prod_{r=1}^{\varrho} \delta_{i_{r}} \prod_{r=1}^{\varrho} \\
& \times\left.\nabla_{\imath_{r}}\left[E\left(u_{1}, \ldots, u_{N}\right) \exp \left(i \sum_{j} \nu_{j} u_{j}\right)\right]\right|_{u_{\jmath}=U},
\end{aligned}
$$

where $\nabla_{j}$ is the gradient with respect to $u_{j}$ and $s$ is the superficial degree of divergence of $\Gamma$. For $\varrho=0$ the $i_{r}$-sums and -products are not present. Definition (4.3) applies to $\Gamma$ in a $T^{+}$sector, while in $T^{-}$sectors the signs of the two exponents are reversed. The $\Gamma$-subtracted integrand becomes

$$
I\left(u_{1}, \ldots, u_{N}\right)\left\{E\left(u_{1}, \ldots, u_{N}\right)-T_{\Gamma} E\left(u_{1}, \ldots, u_{N}\right)\right\} .
$$

Note that the exponents $\sum \nu_{\imath} u_{\imath}$ and $\sum \mu_{i}^{\varrho} u_{i}$ vanish at $u_{1}=\ldots=u_{N}=U$. A simple calculation shows that the curly bracket in (4.4) vanishes at $u_{1}=\ldots=u_{N}$ of order $s+1$ - provided that all derivatives of $E$ up to order $s$ exist - which suffices to reduce the singularity of the integrand to an integrable strength. This argument is 
only of heuristic value, because $E$ is in fact a singular function. That our prescription nevertheless achieves its purpose is seen by Fourier transforming it and showing that in $p$-space it becomes the usual BPHZ prescription. For simplicity we carry out this transformation formally, term by term, even though the intervening integrals exist only for the sum $\left(1-T_{\Gamma}\right) E$. We write, considering the $T^{+}$case:

$$
\begin{aligned}
E\left(u_{1}, \ldots\right) & =\int \prod_{i} d p_{\imath} \exp \left(i \sum p_{\imath} u_{\imath}\right) \tilde{E}\left(p_{1}, \ldots\right) \\
& =\int \prod d p_{i} \exp \left(-i \sum p_{i} u_{i}\right) \tilde{E}\left(-p_{1}, \ldots\right), \\
I\left(u_{1}, \ldots\right) & =\int \prod d q_{i} \exp \left(i \sum q_{i} u_{i}\right) \delta^{4}\left(\sum q_{i}\right) \hat{I}\left(q_{1}, \ldots, q_{n}\right) .
\end{aligned}
$$

The $\delta^{4}$ factor in the last equation is present because $I$ is translation invariant. The function $\hat{I}$ is only defined on the plane $\sum q_{i}=0$.

The integrated unsubtracted term in the expression (4.4) becomes

$$
(2 \pi)^{4 N} \int \prod d p_{i} \tilde{E}\left(\ldots,-p_{\imath}, \ldots\right) \delta^{4}\left(\sum p_{\imath}\right) \hat{I}\left(\ldots, p_{\imath}, \ldots\right) .
$$

The subtraction term of order $\varrho$ becomes after integration over the $u_{\imath}$ :

$$
\begin{aligned}
S_{\varrho}= & \int \prod_{i} d u_{i} \prod_{r} \delta_{i_{r}}\left[\prod_{r} \nabla_{i_{r}} \int \prod_{\imath} d p_{i} \exp \left(-i \sum \hat{p}_{\imath} u_{\imath}\right) \tilde{E}\left(\ldots,-p_{\imath}, \ldots\right)\right]_{u_{\imath}=U} \\
& \times \int \prod_{\imath} d q_{\imath} \exp \left(i \sum \hat{q}_{i} u_{i}\right) \delta^{4}\left(\sum q_{i}\right) \hat{I}\left(\ldots, q_{i}, \ldots\right)
\end{aligned}
$$

with $\hat{p}_{i}=p_{i}-\nu_{i}, \hat{q}_{i}=q_{i}-\mu_{i}^{\varrho}$. Carrying out the differentiations $\nabla_{\imath_{r}}$ results in the replacement $\nabla_{\imath_{r}} \rightarrow-i p_{\imath_{r}}$. The integral

$$
\int \prod d u_{i}\left(\sum \delta_{\imath} \hat{p}_{i}\right)^{\varrho} \exp \left(-i U \sum p_{\imath}\right) \exp \left(i \sum \hat{q}_{i} u_{i}\right)
$$

can be carried out to yield

$$
(-i)^{\varrho}(2 \pi)^{4 N}\left[\sum_{i} \tilde{\nabla}_{i}\left(\hat{p}_{i}-\frac{1}{N} \sum_{j} p_{\jmath}\right)\right]^{\varrho} \prod_{i} \delta^{4}\left(\hat{q}_{i}+\frac{1}{N} \sum_{j} p_{j}\right),
$$

where $\tilde{\nabla}_{i}$ is the gradient with respect to $q_{i}$. We insert this result in $S_{\varrho}$ and move the $q$-differentiations onto $\delta^{4}\left(\sum q_{i}\right) \hat{I}$ through integration by parts. The terms containing derivatives of $\delta^{4}$ vanish, because $\tilde{\nabla}_{i} \delta^{4}\left(\sum q_{j}\right)$ is independent of $i$ and $\sum_{i}\left(\hat{p}_{i}-\frac{1}{N} \sum_{j} \hat{p}_{\jmath}\right)=0$. Hence all $\tilde{\nabla}_{i}$ act on $\hat{I}$, and using

$$
\delta^{4}\left(\sum q_{i}\right) \prod \delta^{4}\left(\hat{q}_{i}+\frac{1}{N} \sum p_{j}\right)=\delta^{4}\left(\sum p_{\imath}\right) \prod_{\imath} \delta^{4}\left(\hat{q}_{\imath}\right)
$$

we obtain

$$
\begin{aligned}
S_{\varrho}= & (2 \pi)^{4 N} \int \prod d p_{i} \tilde{E}\left(\ldots,-p_{\imath}, \ldots\right) \delta^{4}\left(\sum p_{\imath}\right) \\
& \times\left.\left[\sum\left(p_{i}-\nu_{i}\right) \tilde{\nabla}_{\imath}^{\natural}\right]^{\varrho} \hat{I}\left(\ldots, q_{i}, \ldots\right)\right|_{q_{\imath}=\mu_{i}},
\end{aligned}
$$


which is precisely the BPHZ prescription. Notice that $\sum\left(p_{\imath}-\nu_{i}\right)=0$, so that $\sum\left(p_{i}-\nu_{i}\right) \tilde{\nabla}_{i}$ is a derivation within the plane $\sum q_{i}=0$ where $\hat{I}$ is defined.

If a forest contains nested renormalization parts, e.g. $\Gamma^{\prime} \subset \Gamma$, the subtractions are carried out starting from the outside, i.e. $T_{\Gamma}$ is carried out before $T_{\Gamma^{\prime}}$, in contradistinction to the $p$-space prescription. That this is correct can be seen by an easy extension of the calculation given above.

A nice feature of this $x$-space subtraction scheme is the completely symmetrical way in which the variables $u_{\imath}$ enter: there is no equivalent of the routing problem of momenta in $p$-space.

We must now show that renormalization does not destroy the properties of $\mathscr{W}_{\sigma}$ demonstrated for the unrenormalized expressions in the preceding section. This is a problem only for the proofs which rely in an essential way on the local structure of the integrands, i.e. Ostendorf's proof of the splitting relation (1.8) and the proof of Lemma 3.1.

Let $\Gamma$ be a maximal renormalization part in a $T^{ \pm}$sector of a given $G$-forest, with external variables $u_{1}, \ldots, u_{N}$. Let $E\left(u_{1}, \ldots, u_{N}\right)$ be the integrand of $G_{\Gamma}$, as described above. The subtraction terms coming from $\Gamma$ are of the form

$$
D E(U, \ldots, U) F_{\Gamma}^{ \pm}\left(u_{1}, \ldots, u_{N} ; z_{1}, \ldots, z_{M}\right)
$$

The $z$ are the variables of the internal (= not directly connected to $G_{\Gamma}$ ) vertices of $\Gamma, D E$ is a $u_{i}$-derivation of $E, U$ is a linear combination of the $u_{\imath}$ and possibly the $z_{j}$, and $F_{\Gamma}$ is the part of the $G$-integrand coming from $\Gamma$, including the factors $\delta_{\imath_{r}}$ of formula (4.3). The factor $D E$ has the form of a conventional integrand of the reduced graph $G_{\Gamma}^{\prime}$, which results from $G$ by collapsing $\Gamma$ to a point, except that the propagators joining this point may be differentiated and the vertex factor of the collapsed point has the complicated form $F_{\Gamma}^{ \pm}$. The proofs in question would be applicable to $D E$ if $F_{\Gamma}^{-}=-F_{\Gamma}^{+}$were true. This is not the case for the non-integrated form. But it can be shown that $F_{\Gamma}^{-}$may be replaced by $-F_{\Gamma}^{+}$without changing the value of the final integration over the internal variables of $G$. This is easier to see in $p$-space than in $x$-space. Let the corresponding expressions be $F_{\Gamma}^{ \pm}\left(p_{1}, \ldots, p_{N} ; q_{1}, \ldots, q_{R}\right)$. They are given by Feynman graphs $\Gamma$ of the $T^{ \pm}$type, with $p_{\imath}$ as the external variables corresponding to the $u_{i}$, and $q_{j}$ the momenta of the internal lines of $\Gamma . F_{\Gamma}^{ \pm}$may possibly be a derivative with respect to the $p_{i}$ of an ordinary Feynman integrand, and it is to be evaluated at $p_{i}=\mu_{i}^{\varrho}$. The integrand may contain subtraction terms from renormalization parts $\Gamma^{\prime} \subset \Gamma$. We assume that for these lower-order subtractions our contention has already been proved. Under this inductive assumption we can apply Lemma 3.1 and obtain

$$
\begin{aligned}
& F_{\Gamma}^{+}\left(\mu_{1}^{\varrho}, \ldots, \mu_{N}^{\varrho} ; q_{1}, \ldots, q_{R}\right)+F_{\Gamma}^{-}\left(\mu_{1}^{\varrho}, \ldots ; \ldots, q_{R}\right) \\
& \quad=-\sum F_{L R}\left(\mu_{1}^{\varrho} \ldots ; \ldots, q_{R}\right)
\end{aligned}
$$

where the sum extends over mixed graphs with a non-empty $T^{+}$sector $\Gamma_{L}$ and a nonempty $T^{-}$sector $\Gamma_{R}$. These mixed integrands vanish by momentum conservation, because the momenta of the lines connecting $\Gamma_{L}$ to $\Gamma_{R}$ lie on the positive mass shell, while all partial sums of the external momenta $\mu_{i}^{\varrho}$ are space-like or zero. Hence $F_{\Gamma}^{+}=-F_{\Gamma}^{-}$at the relevant points, which proves our claim. 


\section{Infrared Existence}

In the case $m=0$ we expect to encounter IR singularities, under which heading we also include the collinear singularities. Indeed, the individual graphs contributing to a given $\mathscr{W}$ are in general IR divergent. This is most easily seen in $p$-space. A sector represents a contribution to a $\tau^{+}$or $\tau^{-}$function which is amputated with respect to the variables belonging to lines leading to another sector. These $\tau^{ \pm}$are known to possess logarithmic singularities at the mass shell, hence the product of a sector, integrated over its internal variables, with the adjacent $\delta_{+}$propagators is in general divergent.

We shall show that these divergences cancel between the graphs with the same scaffolding. This proof is simpler to achieve in $x$-space than in $p$-space. In $x$-space IR divergence is not connected with the local singularities of the integrand but with its behaviour at large distances. The propagator functions are for $m=0$,

$$
\Delta_{F}(\xi)=\frac{i}{2 \pi^{2}} \frac{1}{\xi^{2}-i \varepsilon}, \quad \Delta_{+}(\xi)=-\frac{i}{2 \pi^{2}} \frac{1}{\left(\xi_{0}-i \varepsilon\right)^{2}-|\xi|^{2}} .
$$

Along the straight line $\xi=\xi^{\prime}+\lambda n, n$ a non-vanishing 4-vector, these functions decrease for $\lambda \rightarrow \infty$ like $\lambda^{-2}$, except if $n$ is light-like, in which case the decrease is only like $\lambda^{-1}$. This exceptional case is important because it concerns the directions along which the singularity sets of the functions approach infinity. And the neighbourhood of such a singularity can be expected to contribute importantly to the integral.

We shall first show that this exceptional behaviour is indeed responsible for the IR problem, and then that the dangerous region can be avoided in the sum over the graphs with the same scaffolding by a deformation of certain paths of integration into complex paths.

Let $x_{1}, \ldots, x_{n}, n \geq 2$, be the external variables of a connected graph, $u_{1}, \ldots, u_{\sigma}$, its internal variables. The $x_{i}$ are integrated over sufficiently smooth test functions, which we assume for simplicity to have compact support. This is no serious restriction: since the integrand is a rational function, possibly multiplied with simple oscillatory exponentials, it is clear that the $u$-integral, if it exists at all, defines a tempered distribution. For the moment we disregard the exceptional directions mentioned above, i.e. we assume that the propagators decrease of second order in all directions, and that their singularities cause no problems.

Let us study the decay of the integral in a given direction in the $4 \sigma$-dimensional $u$ space, i.e. the behaviour along the line $u_{i}=\hat{u}_{i}+\lambda n_{i}$ for $\lambda \rightarrow \infty$, the $n_{i}$ and $\hat{u}_{i}$ being fixed 4-vectors. We also define such $n_{j}^{\prime} s$ for the external points $x_{j}$ by setting $n_{\jmath}=0$. A set of variables with identical $n_{\imath}$ is called a cluster. Inside a cluster the distances between points remain constant for $\lambda \rightarrow \infty$, so that propagators connecting variables in a cluster do not decrease. All external variables belong to the same cluster. Let $N$ be the number of the other, exclusively internal clusters. Assume at first that there are no internal clusters (called 2-clusters) which are connected to the rest of the graph by only two lines. Then we have $P \geq \frac{1}{2}(2+4 N)$ lines connecting different clusters, and their propagators give a decrease of order $\lambda^{-2 P}$. There are $N$ independent inter-cluster distances, leading to a $I=4 N$ dimensional integration over unbounded variables. The difference $2 P-I \geq 2$ is positive, which suffices for convergence at infinity.

If 2-clusters are present, the foregoing estimates do not hold. But a 2-cluster consists necessarily of the vertices of a subgraph with only two external lines, called a 
2-subgraph. And the problem can be handled by enlarging our procedure to a two-stop process: in the first step we integrate over the internal variables of all 2-subgraphs, including all the necessary terms which make these integrations both UV and IR finite. In the second step we apply the previous proof to the remaining variables. More concretely, this means the following. Let $u, v$, be the external variables of a 1-particle irreducible 2-subgraph, $u_{1}, \ldots, u_{\tau}$, its internal variables. If $u$ and $v$ belong to the same sector this holds also for the $u_{\imath}$, and the subgraph is a renormalization part in the sense of Sect. 4 . We must then take it together with the subtraction terms and finite renormalizations as explained there. Since the external lines of a 2-point renormalization part cannot be external lines of a larger renormalization part, we do not get into conflict with the outside-first ordering rule for subtractions by integrating first over $u_{i}, u, v$. If $u$ and $v$ belong to different sectors, we must sum over all sector partitions of the subgraph which leave the $u-v$-assignments intact. We make the inductive assumption that this guarantees IR convergence of the subgraph. Call the integral over the internal variables $C(u, v)$. From Sect. 4 we know that its Fourier transform $\tilde{C}(p, q)$ is of the form

$$
\tilde{C}(p, q)=\delta^{4}(p+q) p^{2} \tilde{F}(p)
$$

where $\tilde{F}$ may be logarithmically divergent at $p^{2}=0$, but not stronger. In $x$-space this becomes

$$
C(u, v)=\square F(u-v)
$$

with $F(\xi)$ decreasing as $|\xi|^{-4}$ for $|\xi| \rightarrow \infty$, possibly up to a power of $\log |\xi|$. We multiply $C$ with the adjacent propagators, which decrease like $|u|^{-2}$ and $|v|^{-2}$ respectively. The d'Alembertian $\square$ can be moved over to these propagators through integration by parts. Then either one of the propagators decreases of fourth order, or both of third order, and this stronger decrease offsets the reduction of $P$ in our earlier estimate of the degree of convergence of the full graph. The generalization of this argument to 1-particle reducible 2-clusters is immediate.

As a side remark we note that this proof is generalizable to other renormalizable theories without superrenormalizable couplings. E.g. in Yang-Mills theories the presence of 3-prong vertices invalidates our estimate for $P$, but this is offset by the fact that at least one of the propagators joining such a vertex is differentiated and therefore decreases of third order at infinity.

We have demonstrated that our graphs would be IR convergent if the propagators decreased like $\lambda^{-2}$ in all directions. Therefore their factual divergence must be due to the slower decrease in light-like directions, It remains to be shown that these directions can be avoided in the sum over all sector partitions of a given scaffolding.

Let again $X=\left\{x_{1}, \ldots, x_{n}\right\}$ be the external, $U=\left\{u_{1}, \ldots, u_{\sigma}\right\}$ the internal variables of a graph. We consider the integrand at fixed values of the $x_{i}$ (or integrated over test functions with compact support, in order to avoid sitting exactly on a $X$ singularity), so that we can find a positive constant $A$ with

$$
\left|x_{i}^{0}\right| \leq A
$$

for all $x_{i}$. Since the IR problems are due to large light-like $z_{i}-z_{j}$, it is useful to decompose the region of integration into suitable smaller parts. For each part there should exist a partition $U=U_{0} \cup U_{+} \cup U_{-}$such that the time components of $U_{0}$ variables are confined to a compact interval $[-B, B]$, those of $U_{+}$or $U_{-}$variables to $\left\{u_{\imath}^{0} \geq B\right\}$ and $\left\{u_{i}^{0} \leq-B\right\}$ respectively. To this end we introduce $C^{\infty}$ functions 
$\alpha_{+}^{A}(\xi), \alpha_{-}^{A}(\xi), \beta^{A}(\xi)$, with supports in $[A, \infty],[-\infty,-A],[-2 A, 2 A]$ respectively, such that

$$
\alpha_{+}^{A}(\xi)+\alpha_{-}^{A}(\xi)+\beta^{A}(\xi) \equiv 1 .
$$

Define

$$
\gamma_{ \pm}^{A}(\xi)=1-\alpha_{ \pm}^{A}(\xi)
$$

We multiply our integrand with the decomposition of the identity

$$
1=\sum_{i=1}^{\sigma}\left[\alpha_{+}^{A}\left(u_{i}^{0}\right)+\alpha_{-}^{A}\left(u_{i}^{0}\right)+\beta^{A}\left(u_{\imath}^{0}\right)\right]
$$

and pick out the contribution of an arbitrary term in this decomposition. If this term contains only $\beta^{A}$ functions, then in its support all $u_{i}$ are restricted to the set $u_{\imath}^{0} \in[-2 A, 2 A]$, i.e. the $u_{i}^{0}$ integrations extend over a compact set only. In this case we define $U_{0}=U, U_{ \pm}=\emptyset$. If the chosen term contains no $\beta$ factors, we define $U_{0}=\emptyset$ and $U_{ \pm}$as the sets of the $u_{i}$ occurring in $\alpha_{ \pm}$factors. The $u_{i}^{0}$ integration extends then only over $u_{i}^{0} \geq A$ or $u_{i}^{0} \leq-A$ respectively. A mixed term we decompose further with respect to its $\alpha$-variables. We define $U_{0}^{(1)}$ as the set of the $u_{\imath}$ in $\beta^{A}$ factors, $U_{ \pm}^{(1)}$ as the sets of $u_{i}$ in $\alpha_{ \pm}^{A}$ factors, and we multiply the term with

$$
1=\prod_{U_{+}^{(1)}}\left[\alpha_{+}^{2 A}\left(u_{i}^{0}\right)+\gamma_{+}^{2 A}\left(u_{\imath}^{0}\right)\right] \prod_{U_{-}^{(1)}}\left[\alpha_{-}^{2 A}\left(u_{\imath}^{0}\right)+\gamma_{-}^{2 A}\left(u_{i}^{0}\right)\right] .
$$

Again we treat each term in this expansion separately. In the term containing only $\gamma$-factors all the $u_{i}$ are restricted to $u_{i}^{0} \in[-4 A, 4 A]$. We define $U_{0}=U, U_{ \pm}=\emptyset$. In the term only containing $\alpha$ 's we define $U_{0}=U_{0}^{(1)}, U_{ \pm}=U_{ \pm}^{(1)}$, and our requirement is satisfied for $B=2 A$. In the mixed $\alpha$ - $\gamma$-terms we define $U_{ \pm}^{(2)}$ as the sets of $u_{i}$ 's occurring in $\alpha_{ \pm}^{2 A}$ factors, $U_{0}^{(2)}$ as the complement of $U_{+}^{(2)} \cup U_{-}^{(2)}$ in $U$. With respect to $L_{ \pm}^{(2)}$ we repeat the procedure, splitting as in (5.6) with functions $\alpha_{ \pm}^{4 A}, \gamma_{ \pm}^{4 A}$, and so on. Clearly the procedure ends after a finite number of steps. For each term there exists a partition $U=U_{0} \cup U_{+} \cup U_{-}$and a positive constant $B \geq A$ such that in its support we have

$$
\left|u_{i}^{0}\right| \leq B \quad \text { for } \quad u_{\imath} \in U_{0}, \quad u_{i}^{0}\left\{\begin{array}{l}
\geq \\
\leq
\end{array}\right\} u_{j}^{0} \quad \text { for } \quad u_{i} \in U_{ \pm}, \quad u_{j} \in U_{0},
$$

and the $\alpha-\beta$ - $\gamma$-product is independent of $u_{\imath} \in U_{+} \cup U_{-}$if $\left|u_{i}^{0}\right|>2 B$.

The $u_{i} \in U_{0}$ cannot go to infinity in a light-like direction; the singularities in these variables are confined to a bounded region. Hence the IR difficulties are caused by $U_{ \pm}$, and we must show that the integration over those $u_{\imath}^{0}$ can be bent away from the dangerous points $u_{\imath}^{0} \sim \pm\left|\mathbf{u}_{\imath}\right|$ at large distances. For this we need

Lemma 5.1. Let $\mathscr{G}$ be the set of all graphs contributing to $\mathscr{W}\left(X_{1}, s_{1}|\ldots| X_{N}, s_{N}\right)$ with the same scaffolding. Let the internal variables $U$ be partitioned into the sets $U_{0}, U_{+}, U_{-}$, introduced above for a given term in the splittings (5.5-6) applied to all $G \in \mathscr{G}$. Then in the sum of the integrands over $\mathscr{G}$ all terms cancel except those in which $U_{ \pm}$variables are only contained in the extremal sectors $S_{1}, S_{N}$, such that $S_{1}$ contains only $U_{+}\left(U_{-}\right)$variables if $s_{1}=+(-)$, and $S_{N}$ contains only $U_{-}\left(U_{+}\right)$ variables if $S_{N}=+(-)$. 
Proof of Lemma 5.1. Consider any graph $G \in \mathscr{G}$. Let $S$ be one of its sectors, $X^{S}$, $U_{0,+,-}^{S}$ its variables. Let $S\left(X^{S}, U_{0}^{S}, \ldots\right)$ be the contribution of $S$ to the full integrand. Then we have, by the splitting relation (1.8):

$$
\begin{aligned}
& S^{+}\left(X^{S}, \ldots\right)=S^{+}\left(U_{+}^{S}\right) S^{+}\left(X^{S}, U_{0}^{S}\right) S^{+}\left(U_{-}^{S}\right) \\
& S^{-}\left(X^{S}, \ldots\right)=S^{-}\left(U_{-}^{S}\right) S^{-}\left(X^{S}, U_{0}^{S}\right) S^{-}\left(U_{+}^{S}\right)
\end{aligned}
$$

for $S$ a $T^{+}$or $T^{-}$sector respectively. The notation is similar as in Eqs. (3.3) ff: the right-hand sides of (5.8) are understood to contain also the $\Delta_{+}$propagators linking different factors.

Consider first two adjacent external sectors of different type, e.g. $S_{m}^{+} \mid S_{m+1}^{-}$. There are no internal sectors between them. For both sectors we use the decomposition (5.8). Summing over all partitions of $U_{-}^{\prime}=U_{-}^{m} \cup U_{-}^{m+1}$ among the two sectors we find the factor $\sum S^{+}\left(U_{-}^{m}\right) S^{-}\left(U_{-}^{m+1}\right)$, which vanishes according to Lemma 3.1 if $U_{-}^{\prime} \neq \emptyset$. In the case of two adjacent external sectors of equal type, e.g. $S_{m}^{+} \mid S_{m+1}^{+}$, there may occur intermediate internal sectors $S_{\nu}^{+}$with $m<\nu<m+1$. We factorize each sector with $m \leq \nu(S) \leq m+1$ according to Eq. (5.8). Assume that there are sectors with $\nu(S)>m$ with a non-empty $U_{+}$part. Take the right-most factor $S^{+}\left(U_{+}^{\prime \prime}\right), U_{+}^{\prime \prime} \subset U_{+}^{\prime}$. It may either be an internal sector by itself, or it may be part of a larger sector which has been split according to Eq. (5.8). The number of internal sectors in the two cases differs by one, hence the two terms have different sign and cancel each other. Therefore only the terms containing $U_{+}$variables exclusively in $S_{m}^{+}$survive in the sum. In the same way, only the terms with $U_{-}$variables exclusively in $S_{m+1}$ need be taken into account. The proof of the lemma is then obvious.

Take now a graph which survives in the sum of Lemma 5.1. Define the function of $\left\{\mathbf{u}_{i}\right\}$

$$
C=\sum_{U}\left|\mathbf{u}_{\imath}\right|
$$

Assume that the left-most sector $S_{1}$ is a $T^{+}$sector, and let $\left\{u_{1}, \ldots, u_{\tau}\right\}$ be its $U_{+}$ variables. We integrate first over the time components $u_{i}^{0}$ of these variables, for fixed $\mathbf{u}_{\imath}$. These partial integrals are still IR convergent. Partition the domain of integration into subsets defined by a fixed time ordering of the variables. Consider one of these sets, e.g. the simplest:

$$
u_{\imath}^{0} \geq u_{2}^{0} \geq \ldots \geq u_{\tau}^{0} \geq B
$$

We can replace these variables by

$$
\omega_{1}=u_{1}^{0}-u_{2}^{0}, \ldots, \omega_{\tau-1}=u_{\tau-1}^{0}-u_{\tau}^{0}, \quad \omega_{\tau}=u_{\tau}^{0}
$$

as integration variables. We have $\omega_{\alpha} \geq 0$ for $\alpha \leq \tau-1, \omega_{\tau} \geq B$. If $C>B$ we shift the integration path in $\omega_{\alpha}$ in the interval $[2 B, 2 C]$ into the lower half plane of the complex plane: $\omega_{\alpha} \rightarrow \omega_{\alpha}-i v$, with

$$
v_{\alpha}\left(\omega_{\alpha}\right)= \begin{cases}\omega_{\alpha}-2 B & \text { for } 2 B \leq \omega_{\alpha} \leq C+B \\ 2 C-\omega_{\alpha} & \text { for } C+B \leq \omega_{\alpha} \leq 2 C .\end{cases}
$$

This shift is consistent with the analyticity properties of the propagators (5.1). If necessary, the sharp corners of the new paths may be rounded off. The new paths can still be parametrized by the real parts $\omega_{\alpha}$, which necessitates the introduction of the unproblematic factors $\left(1-i v_{\alpha}^{\prime}\right)$. The shift of paths does not change the value of the integral. At the boundary $u_{\alpha}^{0}=u_{\alpha+1}^{0}$ of two different orderings the descriptions 
match smoothly, so that the distribution nature of the integrand does not cause any problems.

If $S_{1}$ is a $T^{-}$sector we proceed analogously with its $U_{-}$variables $\left\{u_{1}, \ldots, u_{\tau}\right\}$. In $u_{1}^{0} \leq u_{2}^{0} \leq \ldots \leq u_{\tau}^{0} \leq-B$ we introduce $\omega_{\alpha}=u_{\alpha}^{0}-u_{\alpha+1}^{0}, \omega_{\tau}=u_{\tau}^{0}$ as new variables of integration and deform the $\omega_{\alpha}$ path in $[-2 C,-2 B]$ into a triangular path in the lower half plane like in the previous case. In the same way we deal with the $U_{+}$or $U_{-}$variables of the right-most sector $S_{N}$. E.g. if $S_{N}$ is a $T^{+}$sector with the $U_{-}$variables $u_{1}, \ldots, u_{\tau}$, we use in $-B \geq u_{1}^{0} \geq \ldots \geq u_{\tau}^{0}$ the variables of integration $\omega_{1}=u_{1}^{0}, \omega_{\alpha}=u_{\alpha}^{0}-u_{\alpha-1}^{0}$ for $\alpha=2, \ldots, \tau$, and integrate in $\omega_{\alpha} \in[-2 C,-2 B]$ over a triangular path lying now in the upper half plane.

After these shifts the estimates given in the first part of this section become applicable. As described there, let the $u_{\imath}$ tend to infinity in the direction $u_{\imath}=\hat{u}_{i}+n_{i} \lambda$, $\lambda \rightarrow \infty$. For sufficiently large $\lambda$, this direction remains within a fixed $U_{0}-U_{+}-U_{-}$ partition. Let $u_{\alpha}$ be a $U_{+}$or $U_{-}$variable, $u_{i}$ an arbitrary variable, such that $u_{\alpha}$ and $u_{i}$ do not belong to the same cluster. This means that $\left|u_{\alpha}^{0}-u_{\imath}^{0}\right|+\left|\mathbf{u}_{\alpha}-\mathbf{u}_{\imath}\right|$ diverges for $\lambda \rightarrow \infty$. If $\left|u_{\alpha}^{0}-u_{\imath}^{0}\right|$ remains bounded, then $\left|\mathbf{u}_{\alpha}-\mathbf{u}_{i}\right|$ diverges, and the distance from the dangerous singularity at $\left|u_{\alpha}^{0}-u_{i}^{0}\right|=\left|\mathbf{u}_{\alpha}-\mathbf{u}_{\imath}\right|$ increases linearly in $\lambda$. Otherwise we find that $C \rightarrow \infty$ in the vicinity of this critical manifold and that for large $\lambda$ we have there $\left|u_{\alpha}^{0}-u_{\imath}^{0}\right| \sim\left|\mathbf{u}_{\alpha}-\mathbf{u}_{\imath}\right| \leq C \ll 2 C$. From this it is easily seen that at the critical points the imaginary part along the shifted integration contour increases linearly in $\lambda$, so that also in this case the distance from the singularity increases linearly. This suffices to make our earlier estimates valid in all directions, which proves the desired IR convergence.

\section{References}

1. Steinmann, O.: Asymptotic completeness in QED II. Nucl. Phys. B 361, 173 (1991)

2. Ostendorf, A.: Feynman rules for Wightman functions. Ann. Inst. H. Poincaré 40, 273 (1984)

3. Streater, R.F., Wightman, A.S.: PCT, Spin \& statistics, and all that. Reading, MA: Benjamin/ Cummings 1978

4. Steinmann, O.: Perturbative QED in terms of gauge invariant fields. Ann. Phys. (NY) 157, 232 (1984)

5. Steinmann, O.: On the characterization of physical states in gauge theories. Ann. Inst. H. Poincaré 51, 299 (1989)

6. Zimmermann, W.: Local operator products and renormalization in quantum field theory. Lectures on elementary particles and quantum field theory. Deser, S., Pendleton, H. (eds.). Cambridge, MA: MIT Press 1971

7. 't Hooft, G., Veltman, M.: Diagrammar. Internal report CERN 73-9, Geneva 1973

8. Itzykson, C., Zuber, J.B.: Quantum field theory. New York: McGraw-Hill 1980 
